Most of the essays are critical reviews rather than comprehensive updates of the literature, and many succeed in describing accurately the root observations of the field and critically evaluating our current understanding of the phenomena thus revealed. For this reason the volume should be an enduring one.

Contributions focus on several themes. One is the analysis of how isolated cells move on artificial substrata. Movement of fibroblasts is especially well treated. The topic of polarization of the moving cell as seen by light microscope cinematography is prominent, and the ultrastructural correlates of the light microscopic image are given in detail. A good deal of attention is also devoted to the small-scale specializations of the cell surface which seem to be the actual sites of adhesion, namely focal adhesion plaques and close contact zones. The question of which attributes of artificial substrata are necessary for cell movement and adhesion is reviewed in several of the essays, although the problem of the mechanisms which generate the force necessary for cell motility is not extensively treated. The initial interest in fibroblast movement has since been extended to other types of cells, most notably nerve and epithelia, so it is appropriate that three excellent essays deal with the question of nerve growth cone motility and two with epithelia.

A second theme is the reactions that occur when moving cells in tissue culture encounter one another. Abercrombie's essay reviews the original discovery of contact inhibition between cultured embryonic heart fibroblasts and subsequent efforts to analyse the mechanistic basis for this crucial interaction. The theme of cell-cell interactions is carried over to the interactions of nerve cells with each other and of fibroblasts with epithelial cells, the emphasis being on the morphological complexity of such interactions. The natural stage for cell movement and interactions is the organism; cell and tissue movement in embryonic development is particularly well reviewed. Even better is the account of the migration of cells from the neural crest and of entire tissues during gastrulation, topics which receive a clearer and more comprehensive treatment here than any $I$ have seen in recent years.

This is a valuable book because it gives a clear picture of the current intellectual status of an important part of cell biology and embryology. Part of its message is that there is a wealth of interesting facets of cell behaviour which are accessible to investigation. Armed with all the new probes developed over the past few years (monoclonal antibodies, cloned genes, etc.), molecular biologists will be able to read this book and pick their next set of problems.

David I. Gottlieb is in the Department of Anatomy and Neurobiology, Washington University School of Medicine, St Louis.

\title{
Ordered and disordered systems in order
}

\section{Nicolaas Bloembergen}

Nuclear Magnetism: Order and Disorder. By A. Abragam and M. Goldman. Pp.626. ISBN 0-19-851294-5. (Oxford University Press: 1982.) $£ 45, \$ 69$.

PROFESSOR Abragam has scored for the third time in 20 years, thus completing his hat trick. The assist in this case is credited to Dr Goldman. The sport to which this feat applies is the widely diversified field of magnetic resonance; the goals are major publication events scored on the home grounds of the Clarendon Press, Oxford.

Abragam published his first book, The Principles of NuclearMagnetism, already a classic, in 1961. About a decade later, in 1970, he co-authored Electron Paramagnetic Resonance of Transition Ions with Professor Bleaney. The present book follows some ten years after the second one. Aficionados of magnetic resonance will not be disappointed. It is a different ball game in the 1980 s and there is virtually no duplication with the earlier volumes, amongst which Dr Goldman's Spin Temperature and Nuclear Magnetic Resonance in Solids (Clarendon, 1970) should be included. In fact, both this last book and Abragam's Nuclear Magnetism are almost required reading before the present volume can be enjoyed. It is definitely not for beginning students.

The first chapter is called "Epitome of the Theory of Spin Temperature" and is a condensation of Goldman's book, with a concomitant high density of equations. It contains the groundwork for all subsequent chapters, which may be read almost independently of one another.

These remaining chapters fall into two parts. In the first the authors discuss three topics, in which they describe themselves as observers rather than active participants. One is concerned with high resolution spectroscopy in solids. The clever manipulation of nuclear spin systems by pulse sequences and by (de)magnetizations in rotating frames of reference leads to uncanny reversibilities, which defy the application of statistical mechanics to nuclear spin hamiltonians in certain cases. The other two topics, concluding the first half of the book, are nuclear magnetism of solid and liquid $\mathrm{He}_{3}$, respectively. The authors have digested the complex literature about these intriguing quantum solids and liquids, and provide a good introduction to the appreciation of the

\section{New in paper}

Douglas Heggie's Megalithic Science (reviewed in Nature 296, 373; 1982) has now appeared in paperback. Published by Thames and Hudson, the book costs $£ 5.95$. Also new in a paper edition is Genesis by John Gribbin. Publisher is Oxford University Press, price £3.95; for review see Nature 294, 491; 1981. fundamental role that nuclear magnetic resonance (NMR) has played in the development of this important sub-field of low temperature physics. The large body of NMR work on solid hydrogen and methane is not discussed, because of the wish of the authors "to complete a book of finite size in a finite time". Although the temperature may be in the order of milliKelvins in some cases, the nuclear spin systems are still largely disordered, as the Boltzmann energy $k T$ remains large compared to the nuclear spin interactions. Nonetheless, in this first part of the book the authors have put a lot of order into the discussion of these disordered systems.

The second part deals with the ordered systems, where the degree of nuclear spin polarization is significantly different from zero, and may approach unity in some cases. The authors have been leaders in a laboratory group that has made most important contributions to this field, so here they perform as actors instead of spectators. They describe the changes in lineshapes, entropy, susceptibilities and other thermodynamic quantities, when the degree of nuclear spin ordering becomes significant. The method of dynamic polarization is discussed in detail: neutrons are scattered from ordered nuclear spin systems, and neutron spin resonance is induced by the pseudomagnetic interaction with the precessing nuclear spin polarization. Again, enough background is provided for the non-specialists in neutron physics.

The final chapter deals with the antiferromagnetic and ferromagnetic ordered arrangements in nuclear spin systems. With the exception of solid $\mathrm{He}_{3}$ (and nuclear spins in heavy elements, which are not discussed), the ordering is determined by a pure dipolar interaction. This chapter should also be of considerable interest to those who work with the more familiar electronic ferro- and antiferromagnetic materials. The NMR data provide illuminating examples for those more technical fields of magnetism.

In this book a close contact between theory, experiment and data is maintained at all times. The high density of equations is in many spots alleviated by glimpses of physical insight. One would, however, look in vain for detailed descriptions of experimental apparatus.

There is a lot of physics to enjoy in this book. It belongs in all major physics research libraries, as well as in the individual libraries of low temperature physicists and of those active in NMR research or in magnetism. If you enjoyed Abragam and you liked Goldman, you will love Abragam and Goldman.

Nicolaas Bloembergen is Gerhard Gade University Professor at Harvard University. 